\title{
Link Adaptation on Aggregated TVWS Channels
}

\author{
Zinan Lin, Alpaslan Demir \\ InterDigital Communications LLC \\ Melville, NY 11747, USA \\ \{zinan.lin, alpaslan.demir\}@interdigital.com
}

\begin{abstract}
The availability of Television White Space (TVWS) [1] has attracted considerable attention in industry and academia. Given the relatively small amount of bandwidth allocated to each TVWS channel (e.g., 6, 7 or $8 \mathrm{MHz}$ ), and the non-contiguous nature of the allocation, channel aggregation ([2], [3]) is an attractive way to make full use of the available TVWS spectrum and to achieve higher throughput. In this paper we propose and analyze several link adaptation schemes in the context of channel aggregation. We first analyze the SINR conditions of multiple aggregated channels using a model of a modified 802.11n system to support TVWS operation. Then, we investigate three different link adaptation schemes and compute their MAC layer throughputs. Our results show that the SINR variance on the aggregated channels can be high, mainly due to the unique characteristics of TVWS spectrum, i.e., different maximum transmit powers imposed on different types of TVWS channels, and significant Digital TV (DTV) out-of-band emissions to adjacent TVWS channels. Both analytical and numerical results show that independent link adaptation based on each channel SINR provides much higher throughput than the single adaptation schemes, where a common rate is applied on the aggregated channels.
\end{abstract}

Keywords - Channel aggregation, TVWS, MAC throughput, link adaptation,

\section{INTRODUCTION}

TVWS channels are the unused portions of spectrum in TV bands for a given location. They are the channels which are not used by the primary users (e.g., DTV or wireless microphone) or the channels freed up by the transition from the analogue to DTV broadcasting for that location. TVWS spectrum, due to its location in the UHF band (e.g., 470-698 $\mathrm{MHz}$ in the USA, 470-790 MHz in UK) offers excellent propagation characteristics and thus the potential to enable long-range wireless access and provide substantial extra capacity [2]. Several technologies have been proposed for TVWS operation, including modified/enhanced versions of existing standards such as WiFi and WiMAX and new technologies designed specifically for TVWS. There are differences between these technologies depending on the applications they target (e.g. broadband access, M2M communications, hotspot, backhaul access, etc.) [5]. In particular, IEEE 802.11af draft [2] defines modifications to 802.11ac MAC and PHY to meet the legal requirements for channel access and coexistence in the TVWS, which allows the TV band devices (TVBDs) to aggregate multiple TVWS channels using the concept of physical layer channel bonding.
The actual coverage/throughput that can be achieved by TVWS networks depends on multiple factors [6]: 1) The allowable transmit power for a given type TVBD device and the given location and channel combination; 2) The height and gain (directionality) of the TVBDs; 3) Operational frequency, i.e., less path loss is expected in the lower part of the UHF band than the upper part; 4) The level of interference and noise that is present on the TVWS channels.

Unlike that of wireless devices operating in other types of spectrum (e.g. 2.4 or $5 \mathrm{GHz}$ ISM band), the interference of TVWS networks comes from two main sources [7]: 1) fixed strong interference from DTV stations; and 2) dynamic interference from peer TVBDs. Measurements [7] show that there could be more than $20 \mathrm{~dB}$ interference variation among TVWS channels which are adjacent and non-adjacent to DTV occupied channels Measurements in [8] indicate that 50\% (or $35 \%$ ) of TVWS channels are adjacent to a DTV station in Europe (or the USA). Hence, in most cases, the aggregated channels used for a single communication contain not only non-adjacent channels but also adjacent channels. Due to the uncoordinated nature of TVWS networks, the interference levels caused by the co-existing secondary users can fluctuate on different channels. Furthermore, the allowable transmit power is changed upon the location of the channel, e.g., up to $4 \mathrm{~dB}$ variation of transmit power per $5 \mathrm{MHz}$ channel on different types of channels (adjacent vs. non-adjacent) according to FCC rules [1].

All these factors mentioned above cause highly changeable SINR levels on different TVWS channels. Thus, link adaptation on aggregated TVWS channels becomes more problematic. However, most of the existing research and/or implementation is primarily focused on how TVBD transmission impacts the DTV performance (e.g., [9], [10], [11]) or simply assumes that similar SINRs are experienced on available TVWS channels (e.g., [3] and [12]). Therefore, from the TVWS network design standpoint, it would be highly beneficial to investigate how rates should be adapted on multiple aggregated TVWS channels to accommodate the varied channel conditions and what is the throughput that can be achieved by the optimal link adaptation method.

The contribution of this paper is four-fold:

1) We model a TVWS network with WiFi deployment, aggregating multiple TVWS channels with different transmit powers and interference levels. We assume MAC layer aggregation in our analysis and illustrate the benefits. 
2) We analyze SINR values on multiple aggregated channels and illustrate the selected modulation and coding set (MCS) per channel basis.

3) We formulate the MAC layer throughput of multiple link adaptation mechanisms over aggregated TVWS channels and compare their performance.

4) We propose the optimal power allocation mechanism among aggregated channels and specify the advantages of the optimal link adaptation.

This analysis facilitates the design of an optimal TVWS network to achieve a higher throughput without incurring significant complexity and gives an operational guideline to link adaptation and power allocation among aggregated channels.

The rest of this paper is organized as follows. The system model is presented in Section II, which illustrates the TVWS network deployment and the aggregation approach we adopt. Section III analyzes the TVWS network performance that includes SINR calculation and throughput analysis. The numerical results are provided in Section IV, which compares the throughputs of different link adaptation methods and proposes the transmit power allocation mechanism. Section V concludes this paper and discusses the future work.

\section{SYSTEM MODEL}

We consider a simplified TVWS network (Figure 1) deployed with two TVBDs. All nodes are able to operate on TVWS channels. The detailed device architecture to support the TVWS operation is out of scope of this paper and discussed in [3].

To simplify our analysis, we assume the following for the TVWS network deployment: 1) The TVWS network is 802.11n enabled; 2) Each node is installed with one transmit antenna and two receiver antennas, i.e., $1 \mathrm{Tx}$ and $2 \mathrm{Rx} ; 3$ ) Each node is half-duplex, i.e., cannot transmit and receive at the same time; 4) Channel $(\mathrm{CH}) \mathrm{N}$ is occupied by a DTV, $\mathrm{CH}$ $\mathrm{N}+1$ to $\mathrm{CH} \mathrm{N+4}$ are available for secondary usage for the given location; 5) The TVBDs in Figure 1 gain the access to $\mathrm{CHs} \mathrm{N}+1$ and $\mathrm{N}+4$ and perform MAC layer aggregation over these two channels.

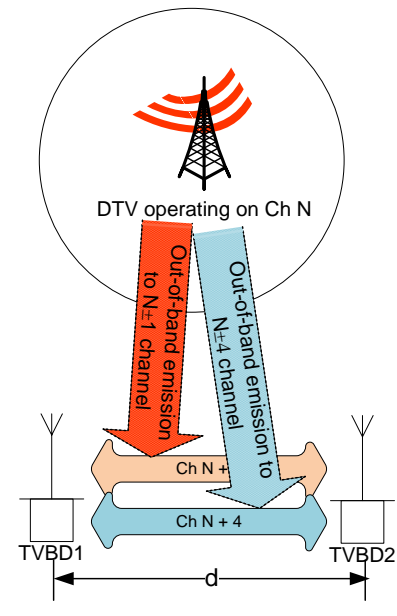

Figure 1 Exemplary Allocation of TVWS channels

\section{A. MAC layer aggregation over multi-TVWS- channel}

Now we briefly illustrate the principle of MAC layer aggregation and its advantages.

MAC layer aggregation enables simultaneous transmissions of multiple MAC service data units (MSDUs) over multiple available physical channels. To avoid the selfinterference among multiple channels and improve the channel access efficiency, we employ the following mechanisms in the MAC layer aggregation:

1) Primary CSMA - One channel is selected as the primary channel in the channel aggregation. The device which wins the CSMA contention on the primary channel gets access to all aggregated channels. It is only when the primary channel is busy that the entire transmission is deferred to a subsequent transmission opportunity.

2) Block ACK - Due to the constraint of half-duplex capability, the acknowledgements need to be synchronized over aggregated channels. One of the approaches shown in [3] is to synchronize ACK transmissions on the aggregated channels. To further improve the MAC efficiency, we use the block ACK protocol ([13]) in the MAC layer aggregation. That is, when a STA wins a transmission opportunity (TXOP), it continuously transmits a block of data frames without requesting ACK until the end of one TXOP. The block ACK (BA) is sent upon the receipt of Block ACK Request (BAR) from the transmitter. The inter-frame space is reduced to RIFS. The exemplary MAC layer aggregation of 2 TVWS channels with primary CSMA and block ACK mechanisms is illustrated in Figure 2. 


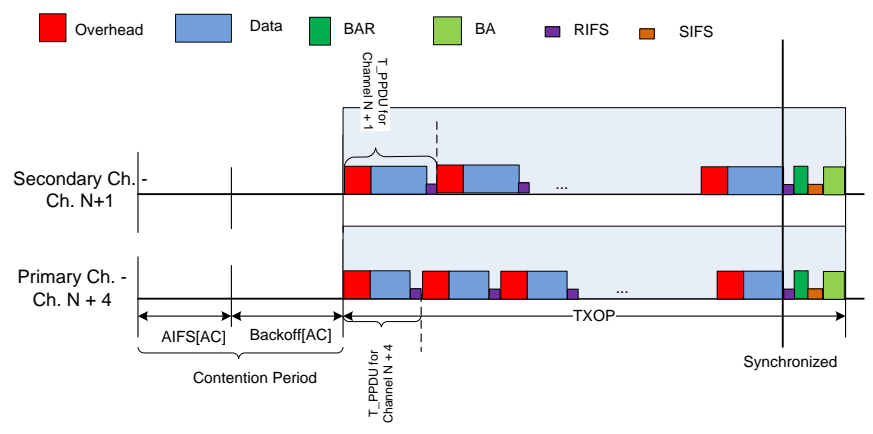

Figure 2 MAC layer aggregation of 2 TVWS channels with primary CSMA and block ACK mechanisms

MAC layer aggregation allows contiguous or noncontiguous channels to be bonded for one-time transmission. When compared with PHY channel bonding employed in 802.11af [2], MAC layer aggregation supports independent PHY operation on each aggregated channel, which enables separate encoding/decoding processes, independent interleaving and MCS to match the SINR of each channel. This feature gives the flexibility of independent link operation on each channel and leads to the potential throughput maximization with the existing PHY design.

\section{PERFORMANCE ANALYsis}

In this section, we analyze the TVWS network performance using MAC layer aggregation. The definitions given in this paper are summarized as follows:

- $\quad \gamma_{k}$ : received SINR on channel $k$;

- $\quad \Gamma_{k}$ : throughput on channel $k$;

- $\quad N_{P P D U, k}$ : number of PPDUs per TXOP on channel $k$ with the selected MCS meeting the target PER of channel $k$;

- $\quad T_{k}$ : total time period used for one packet transmission and response on channel $k$;

- $D$ : down clocking factor used for the TVWS channel operation

- $\quad R_{k}$ : selected PHY data rate on channel $k$;

- $\quad P_{k}$ : transmit power on channel $k$;

- $\quad L_{k}^{P}$ : path loss on primary or secondary channels. In our analysis, we ignore the negligible path loss difference between aggregated channels and assume that the path loss of each channel is roughly the same, i.e., $L_{l}=L_{2}=L$;

- $L_{k}^{O}$ : other losses during transmission and reception, e.g., combiner, feeder, body losses, etc;

- $G_{T X}, G_{R X}$ : transmitter/receiver antenna gain;

- $\quad N_{0}$ : noise density on the channel. We assume that the same total noise levels are experienced on the aggregated channels;

- $\quad I_{k}$ : total interference experienced on channel $k$;

- $W$ : channel bandwidth;

- $P_{e}$ : target Packet Error Rate (PER) on each channel.

\section{A. SINR Calculation and Rate Selection}

To find the optimum link adaptation method, we first need to investigate the channel conditions of TVWS spectrum. In this section, we calculate the SINR values of multiple TVWS channels and describe the selected MCS on each channel.
The average SINR on channel $k$ can be expressed as

$$
\gamma_{k}=\frac{G_{T X} G_{R X} P_{k} L_{k}^{P} L_{k}^{O}}{N_{0} W+I_{k}}
$$

The path loss, $L$, depends on the operational environment, frequency and the distance between the transmitter and the receiver. For the same operational environment and frequency, $\gamma_{\mathrm{k}}$ is a function of distance, interference, $I_{k}$, allowable transmission power, $P_{k}$ and antenna gains $\left(G_{T X}, G_{R X}\right)$.

The interference caused by DTV stations varies with the channel's relative position to DTV occupied channels ([6] and [7]) as well as the distance between the TVBD and the DTV station, among other variables. For example, when a high power DTV signal $-28 \mathrm{dBm}$ [14] is present, according to the FCC DTV emission mask [15], the total DTV out-of-bandemission (OOBE) to the first adjacent channel can be up to -86 $\mathrm{dBm}$ (as shown in [16] and [17]). However, the DTV leakage to the $3^{\text {rd }}$ or $4^{\text {th }}$ adjacent channel is much less than the first adjacent channel, i.e., $-150 \mathrm{dBm}$ or below. Therefore, we can conclude that the regulatory imposed DTV spectrum mask could cause significant variation of SINR values on aggregated channels.

For a given operating environment, the MCS selection depends on the target PER and the received SINR. Given an SINR value, the selected MCS on that channel is the highest one of all supportable MCS's to achieve the target PER. To simplify the analysis, we assume slow link adaptation [18]. The selected MCS on each channel is the long term optimal MCS, which uses long term PER as the adaptation reference and is based on the plots of averaged SNR vs. PER in different types of channel models.

Now we shall illustrate the SINRs of two TVWS channels shown in Figure 1 and the selected MCS's per channel SINR. We adopt the 802.11af PHY design for the single TVWS channel [2], which is based on the $40 \mathrm{MHz} 802.11$ ac standard. We use the MCS parameters defined for the single data stream (MCS0 to MCS7 with the data rate from 13.5/D to $135 / \mathrm{D}$ Mbps) and $\mathrm{D}=7.5 . N_{0}(\mathrm{dBm} / \mathrm{Hz})=$ Thermal Noise Power Spectrum Density (PSD) + Noise Figure (NF), where Thermal Noise PSD is $-174 \mathrm{dBm} / \mathrm{Hz}$ and the $\mathrm{NF}$ of the receiver is assumed to be $5 \mathrm{~dB}$. The bandwidth (BW) of a TVWS channel is assumed to be $6 \mathrm{MHz}$ and the equivalent operational $\mathrm{BW}$ is $W=5.33 \mathrm{MHz}$ [2]. The DTV interference to $\mathrm{CH} \mathrm{N+1}$ is assumed to be $-86 \mathrm{dBm}$ and the DTV leakage to $\mathrm{CH} \mathrm{N+4}$ is much lower than the receiver noise floor. We assume that the target PER is $10 \%$ and the operational frequency is $600 \mathrm{MHz}$. We use the path loss model for Channel Model E [18].

The total transmit-power for the portable TVBD is limited to $20 \mathrm{dBm}$. According to the FCC rules [1], the maximum transmit power on the adjacent channel is confined to $16 \mathrm{dBm}$, i.e., $P_{N+1}=16 \mathrm{dBm}^{1}$. Then the remaining transmit-power assigned to $\mathrm{CH} \mathrm{N}+4$ is $P_{N+4}=17.5 \mathrm{dBm}$. No antenna gain is

${ }^{1}$ In the case where the transmit power is limited to $40 \mathrm{~mW}$ per device using a channel adjacent to DTV broadcaster, then the total transmit power is distributed such that $40 \mathrm{~mW}=\mathrm{P}_{\mathrm{N}+1}$ $+\mathrm{P}_{\mathrm{N}+4}$. 
assumed for the portable TVBDs, i.e., $G_{T X}=G_{R X}=0 \mathrm{~dB}$ and $L^{k}{ }_{o}=1 \mathrm{~dB}$. Using (1), we calculate the SINRs on $\mathrm{CH} \mathrm{N+1}$ and $\mathrm{CH} N+4$ over distances from 50 to $200 \mathrm{~m}$ and show them in Figure 3. The MCS selection of each channel is based on the PER vs. SNR figures provided in [19]. We can observe that when the distance between the TVBD and the DTV station is shorter, both channels have high enough SINR values to select the highest MCS. But with the increasing distance and hence path-loss, the variation of selected MCS based on each channel's SINR becomes larger.

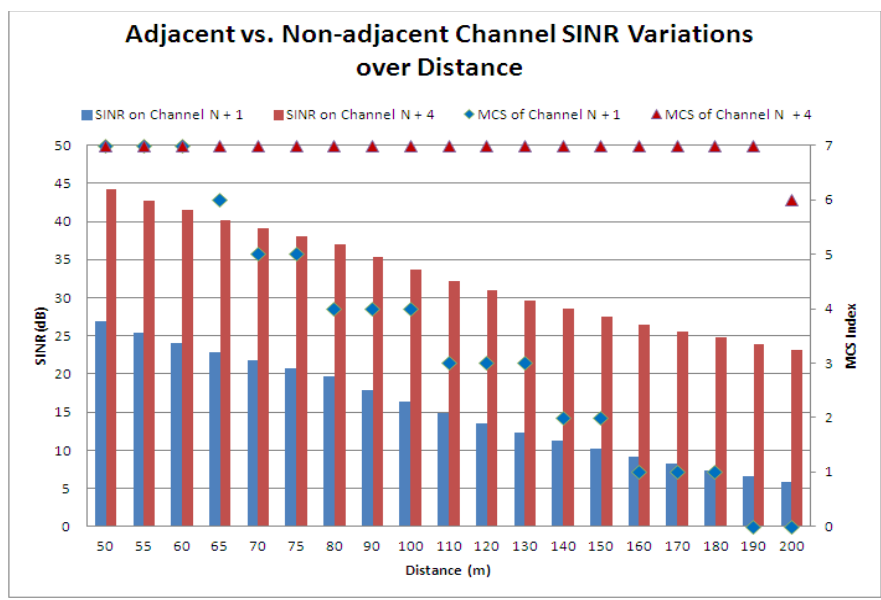

Figure 3 SINR(dB) and MCS Index vs. Distance (m)

\section{B. Throughput Analysis}

Given a selected MCS, we shall formalize the MAC throughput achieved by MAC layer aggregation in this section.

802.11af [2] shows that one PPDU includes three main parts: preamble signals, MAC/IP overhead and data. Then $T_{k}=T_{\text {preamble }}+T_{\text {overhead }, k}+T_{\text {data }, k}$. The time used for preamble transmission, $T_{\text {preamble }}$ is fixed for a given $\mathrm{BW}$ regardless of the channel quality, e.g., $40 \mu$ s for $20 / 40 \mathrm{MHz}$ channel. The transmission time used for data and overhead depends on the selected MCS, which adapts to the channel quality (i.e., SINR). The total transmission time is given as,

$T_{\text {total }}=R I F S+T_{k}=R I F S+T_{\text {preamble }}+\frac{\text { OverheadLength }+ \text { DataLength }}{R_{k}}$

As shown in Figure 2, the time used for transmitting PPDUs within one TXOP equals to TXOP $-B A-B A R-S I F S$. Then the total number of PPDUs transmitted in one TXOP is

$$
\begin{aligned}
& N_{P P D U, k}=\frac{T X O P-B A-B A R-S I F S}{T_{\text {total }}} \\
& =\frac{T X O P-B A-B A R-S I F S}{R I F S+T_{\text {preamble }}+\frac{\text { OverheadLength }+ \text { DataLength }}{R_{k}}}
\end{aligned}
$$

Assuming that unlimited retransmission is allowed, we can easily derive the MAC layer throughput on channel $k$ as

$$
\Gamma_{k}=\frac{N_{P P D U, k} \times \text { DataLength } \times\left(1-P e_{k}\right)}{\text { ContentionPeriod }+T X O P},
$$

where ContentionPeriod $=A I F S[A C]+$ Backoff $[A C]$,
AIFS $[A C]=$ aSIFTime + AIFSN $[A C] *$ aSlotTime and

Backoff $[A C]=\operatorname{random}(0, C W) *$ aSlotTime $[13]$. AISFN [AC] and $\mathrm{CW}$ are the slot count and contention window defined for a particular access category (AC) respectively. $\mathrm{CW} \in\left[\mathrm{CW}_{\mathrm{MIN}}, \mathrm{CW}_{\mathrm{MAX}}\right], \mathrm{CW}_{\mathrm{MIN}}$ and $\mathrm{CW}_{\mathrm{MAX}}$ are dependent on the $\mathrm{AC}$.

Then the total throughput of the TVBDs using MAC layer aggregation is $\Gamma=\sum_{k=1}^{K} \Gamma_{k}$

The parameters that will be used in our analysis are: the lengths of BA and BAR (compressed bitmap) are 24 and 32 bytes, respectively. The AC is Video. It is assumed that the DATA length is 1000 bytes and overhead (including service field, MAC, IP, etc) contains 45 bytes. The preamble time is $300 \mu$ s for a $6 \mathrm{MHz}$ TVWS channel. The MAC parameters are summarized in Table 1:

Table 1 MAC Parameters ( $\mu \mathrm{s})$

\begin{tabular}{|c|c|c|c|c|c|}
\hline TxOP & SlotTime & SIFS & RIFS & AIFS[VI] & $\begin{array}{c}\text { Average } \\
\text { Backoff[VI] }\end{array}$ \\
\hline 23,500 & 67.5 & 120 & 15 & 255 & 596.25 \\
\hline
\end{tabular}

\section{Throughputs Comparison}

As we can see from (2) and (3), MAC layer throughput strongly depends on the selected PHY rate, i.e., MCS. Common MCS for all aggregated channels (as proposed in [2]) would be the simplest approach to the implemented link adaptation. However, due to the highly fluctuating SINRs on multi-TVWS-channel (as shown in Figure 3), it would be desirable to have independent MCS selection to match the SINR of each channel such that the total throughput can be maximized. In this section, we shall analyze the throughputs of three different link adaptation methods and quantize the maximum gain which can be achieved by the independent MCS selection.

Three link adaptation methods we investigate $\left.\operatorname{are}^{2}: 1\right)$ Independent MCS selection (IndMCS): MCS selection based on each channel SINR; 2) Max MCS selection (MaxMCS): the highest MCS among aggregated channels is applied on all channels; 3) Min MCS selection (MinMCS): the lowest MCS among aggregated channels is applied on all channels.

MCS1 and MCS2 refer to the selected MCS's for CH1 and $\mathrm{CH} 2$ respectively per target PER on each channel. Without loss of generality, we assume that $\gamma_{1}<\gamma_{2}$. As what we observe in Figure 3, when the difference between $\gamma_{1}$ and $\gamma_{2}$ is larger than a certain value, the values of MCS1 and MCS2 are different, i.e., $R_{1}<R_{2}$. Using (2), we have $N_{P P D U, 1} \leq N_{P P D U, 2}$. In the following theoretical analysis, we also assume that given the selected MCS per target PER, the resulting PER on each channel is same as the target PER (although in the real

${ }^{2}$ Due to the paper space constraint, we choose two typical single adaptation mechanisms, MaxMCS and MinMCS to compare with IndMCS in this paper. 
implementation the actual PER caused by the selected MCS could be slightly different from the target one). We define $P e_{12}$ is the PER of CH1 with MCS2 applied and $P e_{2 l}$ is the PER of $\mathrm{CH} 2$ with MCS1 applied.

Then the respective total throughput of two aggregated channels using IndMCS, MaxMCS and MinMCS are given below:

$$
\begin{gathered}
\Gamma_{\text {Ind }-M C S}=\frac{\left(N_{P P D U, 1}+N_{P P D U, 2}\right) \times \text { DataLength } \times(1-P e)}{\text { ContentionPeriod }+T X O P}, \\
\Gamma_{\text {Max }-M C S}=\frac{\text { DataLength } \times N_{P P D U, 2} \times\left[\left(1-P e_{12}\right)+(1-P e)\right]}{\text { ContensionPeriod }+T X O P},
\end{gathered}
$$

and

$$
\Gamma_{\text {Min-MCS }}=\frac{\text { DataLength } \times N_{P P D U, 1} \times\left[(1-P e)+\left(1-P e_{21}\right)\right]}{\text { ContentionPeriod }+T X O P} .
$$

Now we compare the throughput of IndMCS with the other two methods:

$$
G_{12}=\frac{\Gamma_{I n d-M C S}-\Gamma_{M a x-M C S}}{\Gamma_{M a x-M C S}}=\frac{\left(N_{P P D U, 1}+N_{P P D U, 2}\right)(1-P e)}{N_{P P D U, 2}\left[(1-P e)+\left(1-P e_{12}\right)\right]}-1
$$

and

$$
G_{13}=\frac{\Gamma_{I n d-M C S}-\Gamma_{M i n-M C S}}{\Gamma_{M i n-M C S}}=\frac{\left(N_{P P D U, 1}+N_{P P D U, 2}\right)(1-P e)}{N_{P P D U, 1}\left[(1-P e)+\left(1-P e_{21}\right)\right]}-1,
$$

where $G_{12}$ and $G_{13}$ define the throughput gain of IndMCS over MaxMCS and MinMCS, respectively. Given $\gamma_{1}<\gamma_{2}$, if MCS2 is used in channel 1, it could cause a higher PER on $\mathrm{CH} 1$ than the target one, i.e., $1 \geq P e_{12} \geq P e$; similarly, MCS1 may result in a lower PER on $\mathrm{CH} 2$ than the target one, i.e., $0 \leq P e_{21} \leq P e$. Given these conditions, we can easily derive that

$$
G_{12} \in\left[\frac{1}{2} \frac{N_{P P D U, 1}}{N_{P P D U, 2}}-\frac{1}{2}, \frac{N_{P P D U, 1}}{N_{P P D U, 2}}\right]
$$

and

$$
G_{13} \in\left[\frac{1+\frac{N_{P P D U, 2}}{N_{P P D U, 1}}}{1+\frac{1}{1-P}}-1, \frac{1}{2} \frac{N_{P P D U, 2}}{N_{P P D U, 1}}-\frac{1}{2}\right] .
$$

As $N_{P P D U, 1} \leq N_{P P D U, 2}, G_{12}$ is always smaller than 1 . We can also easily derive that that $G_{12}$ reaches its maximum when $N_{P P D U, 1} / N_{P P D U, 2}=1$ and $P e_{12}=1$. While the range of $\mathrm{G}_{13}$ depends on the target PER and $N_{P P D U, 2} / N_{P P D U, 1}$. Using the parameters in Table 1, we find that for $P e=0.1, G_{13}$ proportionally increases from 0 to 2.68 with the differentiations between the PHY data rates selected for aggregated channels.

Above comparisons indicate that comparable channel conditions (i.e., $\frac{N_{P P D U, 1}}{N_{P P D U, 2}} \rightarrow 1$ ) and the higher $P e_{1,2}$ cause the higher throughput loss in MaxMCS. However, when channel conditions of both channels are similar, MinMCS results in less performance loss from IndMCS. When the variation of channel conditions is large, MinMCS leads to a significant waste of the spectrum usage and greatly limits the system throughput. Therefore, a possible implementation would be to disable aggregation and allocate the total transmit power to the better channel when the variation of the aggregated channels is larger than a certain threshold. This will also increase the coverage range of the channel. In addition, to ensure the robustness of TVWS operations, it is always beneficial to choose the strongest channel (with the highest average SINR) as the primary channel and the remaining ones are chosen as the secondary channels.

\section{RESULTS AND DISCUSSIONS}

In this section, we first present the throughput performance of three link adaptation mechanisms. Using the optimal link adaptation scheme as reference, we then propose a practical transmit power allocation for the TVWS network deployment. The numerical throughputs of three link adaptation mechanisms are given in Figure 4. Figure 5 compares the throughput gains of IndMCS over MaxMCS and MinMCS, respectively. The throughput gains are consistent with the analytical results given in Section III: $G_{12}$ is always less than 1 and reaches $69 \%$ at the distance $90 \mathrm{~m}$ (less channel variation but higher PER on the weaker channel caused by the MaxMCS), and $G_{13}$ goes up to $240 \%$.

We observe that for larger distance (e.g., $\geq 190 \mathrm{~m} @$ Channel E) the total throughput is dominated by the better channel. Thus, IndMCS offers less benefit to MaxMCS. For MaxMCS, when the distance is larger than $80 \mathrm{~m}$, only the better channel is favorable and the weaker channel is unusable and jammed with wasted transmissions. MinMCS provides much lower throughput due to the conservative selection in larger distance. In high SNIR cases (e.g., distance $\leq 70 \mathrm{~m} @$ Channel E), all selection methods lead to the same MCS selection on both channels and similar PER performance, thus similar throughput.

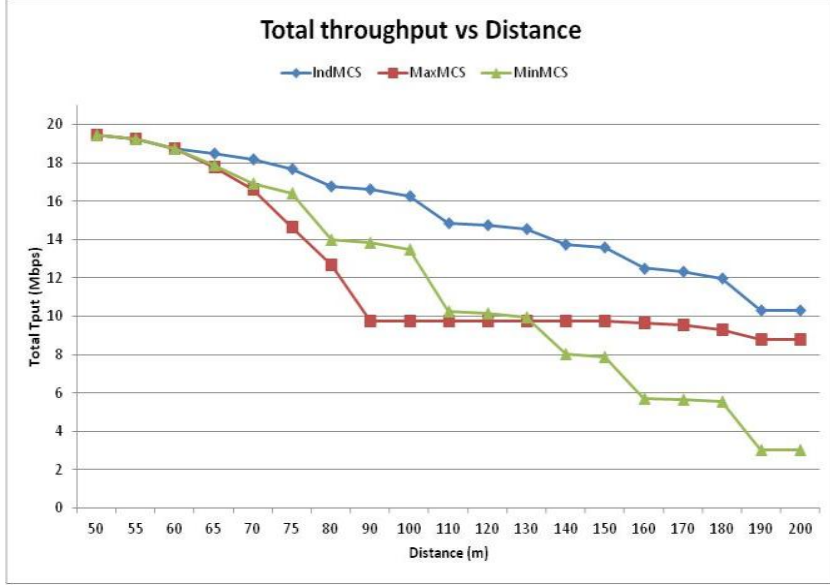

Figure 4 Throughputs of Independent MCS Selection, Max MCS Selection and Min MCS Selection vs. Distance(m) in Channel E 


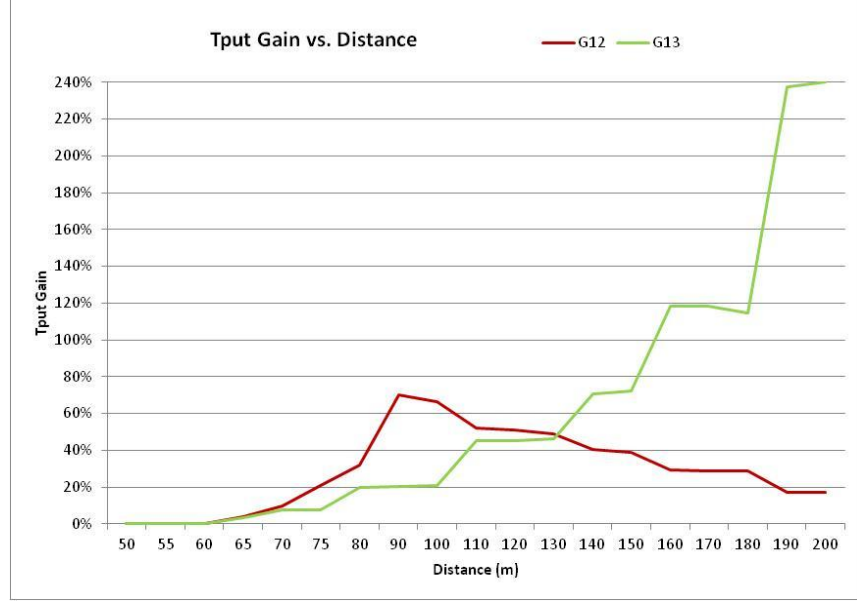

Figure 5 Throughput gains of IndMCS over MaxMCS and MinMCS vs. Distance (m) in Channel E

Figure 6 compares the throughputs of aggregating two TVWS channels with IndMCS and using only the better channel. As discussed in Section III, if the system requirements permit, a simpler implementation that suffers a minimal loss would be to allocate the full transmit power to the better channel beyond a certain distance. For example, to allow up to $10 \%$ throughput loss compared with IndMCS, the turning point in our example (10\% PER, Channel E as shown in Figure 6) is roughly $190 \mathrm{~m}$. That is when distance $\geq 190 \mathrm{~m}$, the active TVBD can use the full transmit-power on the better channel. By doing this, the coverage is increased from $680 \mathrm{~m}$ (with aggregating two channels) to $800 \mathrm{~m}$ (operating on the better channel only).

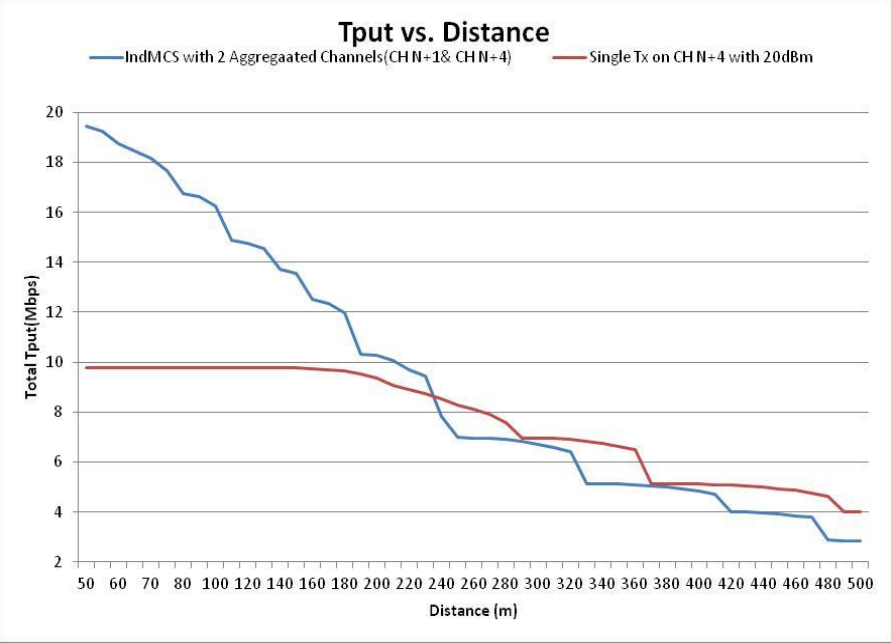

Figure 6 Throughputs of aggregating two TVWS channels vs. using a single better channel for transmission in Channel $\mathrm{E}$

\section{CONCLUSION}

In this paper, the throughput performance of a TVWS network using MAC layer aggregation is analyzed. It shows that the SINR on multiple aggregated TVWS channels varies significantly. Two main factors cause this variation: different received power on different TVWS channels and a highly varying interference environment, especially DTV interference. Results show that a single MCS utilization for multiple channels could lead to significant performance degradation. The IndMCS scheme for multiple channels permits efficient use of resources in real TVWS environments and offers the highest throughput. However, when the variation of aggregated channels is larger than a threshold, the channel with the worst condition can be removed from the aggregation and the transmit power can be fully allocated to the better channel(s) to extend the coverage with no or minimum throughput loss. Next steps include comparing the throughput performance of our prototype (i.e., real system implementation) with the analytical results presented here. Other single adaptation methods may be worth further investigations.

\section{REFERENCES}

[1] Federal Communications Commission, "Third Memorandum Opinion and Order," FCC 12-36A1, April, 2012

[2] IEEE 802.11 af Standard, Part 11: Wireless LAN Medium Access Control (MAC) and Physical Layer (PHY) Specifications, Amendment 5: TV White Spaces Operation, July 2012.

[3] Yuying Dai, Angelo Cuffaro, Jean-Louis Gauvreau, Rocco Di Girolamo, Athmane Touag, Alpaslan Demir, "Dynamic Spectrum Management System Aggregating Non-Contiguous Spectrum over TVWS," presented at $7^{\text {th }}$ CROWN, June 18-20, 2012, Stockholm, Sweden.

[4] Cambridge TV white spaces trial: a summary of the technical findings," 2012 Cambridge White Spaces Consortium.

[5] Rashid, A. Saeed and Stephen J. Shellhammer, TV White Space Spectrum Technologies, CRC Press, 2012.

[6] "Recommendations from implementing the use of white spaces: conclusions from Cambridge TV White Spaces Trials," 2012 Cambridge White Spaces Consortium.

[7] Neul, “Using White Space for M2M," Weightless, June 2011.

[8] Jaap van de Beek, Janne Riihijärvi, Andreas Achtzehn and Petri Mähönen, "TV white space in Europe," IEEE Trans. On Mobile computing, vol. 11, no. 2, pp. 178-188, Feb. 2012.

[9] Gordon. L. Stuber, Sami M. Almalfouh and Darryl Sale, "Interference anlysis of TV-band whitespace," Proceedings of the IEEE, vol. 97, no. 4, April 2009.

[10] Oded Bendov, "Interference to UHF-DTTV channels by unlicensed devices," IEEE Trans. on Broadcasting, vol. 52, no. 4, Dec. 2006

[11] Lei Shi, Ki Won Sung, and Jens Zander, "Controlling aggregate interference under adjacent channel interference constraint in TV White Space," presented at $7^{\text {th }}$ CROWN, June 18-20, 2012, Stockholm, Sweden.

[12] Ljiljana Simi 'c, Marina Petrova and Petri Mähönen, "Wi-Fi, but not on Steroids: Performance Analysis of a Wi-Fi-like network operating in TVWS under realistic Conditions," presented at the ICC, June 10-15, 2012, Ottawa, Canada.

[13] IEEE Part 11: Wireless LAN Medium Access Control (MAC) and Physical Layer (PHY) Specifications, IEEE 802.11-2012.

[14] FCC, "Evaluation of the performance of prototype TB-Band white space devices Phase II," OET 08-TR-1005, Oct. 2008.

[15] A.T.S. committee, "ATS recommendeed practice: Transmission measurement and compliance for digital television," Document A/64B May 2008.

[16] Hou-Shin Chen and Wen Gao, "Spectrum sensing for FM wireless microphone signals," in IEEE DySPAN 2010, Singapore, April, 2010.

[17] InterDigital, "Signal to Noise+Interference (SNIR) Variations on multiple TVWS channels,” IEEE 802.11-12/0924r0, July 2012.

[18] Eldad Perahia and Robert Stacey, Next Generation Wireless LANs Throughput, Robustness, and Reliability in 802.11n, Cambridge, 2010.

[19] “Joint proposal team PHY simulatino results," IEEE 802.11-06/0067r0. 